\title{
WRAN Y LTE EN LA BANDA DE 700 MHz PARA COLOMBIA
}

\section{WRAN AND LTE IN THE 700 MHz BAND FOR COLOMBIA}

\author{
Ing. Fabián Camilo Cárdenas, Ing. Carolina Rivera Sáenz. \\ PhD. Oscar Elías Herrera Bedoya.
}

Universidad Piloto de Colombia, Programa Ingeniería de Sistemas, Grupo InnovaTIC. Carrera 9, No. 45A - 44, Bogotá D.C., Colombia, Tel.: (+571) 3322900.

E-mail: $\{$ fcardenas61, crivera71\} @upc.edu.co, oscar-herrera@ unipiloto.edu.co

\begin{abstract}
Resumen: En el desarrollo de este trabajo se efectúa el análisis de estos estándares para zonas rurales los cuales pueden en gran medida complementar los objetivos trazados por el MinTIC (Ministerio de la Tecnología de la información y las comunicaciones). Para lograr cumplir con esas proyecciones, se están analizando diferentes alternativas, como LTE (Long Term Evolution) que es un estándar de telecomunicaciones comercializado y masificado en varios países y WRAN (Wireless Rural Area Network) estándar nuevo que está diseñado para trabajar conjuntamente con los sistemas ya implementados en las regiones donde se desea utilizar, estas dos opciones serán estudiadas en la banda de los $700 \mathrm{MHz}$.
\end{abstract}

Palabras clave: LTE, WRAN, dividendo digital.

\begin{abstract}
In the development of this work, we do the analysis of these standards for rural areas, which can greatly complement the goals set by the MinTIC. In order to meet these projections are analyzing different alternatives, such as LTE which is a telecommunications standard commercialized and crowded in several countries and WRAN a new standard that is designed to work with the already implemented systems in the regions where they want to use, these two options will be studied in the $700 \mathrm{MHz}$ band.
\end{abstract}

Keywords: LTE, WRAN, digital dividend.

\section{INTRODUCCIÓN}

Si hacemos una retrospectiva de la evolución de las tecnologías móviles en Colombia, se observa que en los últimos 5 años se han realizado grandes avances en pro de conectar todos los municipios de Colombia por medio de internet, ya sea llevando fibra óptica a las cabeceras de los principales municipios, internet satelital, entre otras. Pero, debido a la diversidad en los paisajes de nuestro país, una gran parte del territorio aun no cuenta con servicios de internet.
Uno de los más grandes retos del actual gobierno es poder aumentar la cobertura de las conexiones a este servicio, es por eso que por medio de programas impulsados por el ministerio de las tecnologías como $4 \mathrm{G}$ para todos y el plan vive digital, se han presentado propuestas muy puntuales para lograr esta gran ambición, y por último en este año que recién comienza se esta llevando acabo el despliegue de unas de las tecnologías que podría revolucionar como vemos el internet y las telecomunicaciones en Colombia, $4 \mathrm{G}$ LTE. 


\section{TECNOLOGÍAS MÓVILES}

En la actualidad, debido al auge de tecnologías como la internet y la necesidad de estar siempre conectados a esta red de información, nacen tecnologías móviles como los sistemas de telecomunicaciones basados en diversos estándares que siempre buscaron mejorar la rapidez y la eficiencia de como las personas acceden a los servicios por medio de diversos dispositivos. Tecnologías como CDMA (Code División Múltiple Access), GSM (Global System for Mobile), GPRS (General packet radio service), UMTS (Universal Mobile Telecommunications System), HSPA+ (High-Speed Packet Access) contribuyeron a que hoy en día podamos disfrutar de los servicios de la internet en cualquier parte del planeta de manera ágil y eficiente, pero, debido al constante cambio en las necesidades y la constante búsqueda en el mejoramiento de los servicios móviles, los estándares actuales se están viendo cortos para suplir las necesidades del nuevo mercado.

\subsection{Plan Vive Digital}

En el último estudio realizado y publicado por el MINTIC (Boletín trimestral de las TIC 2013) presentan los datos de evolución en conexiones a internet fijo y móvil en Colombia en los últimos 2 años. Actualmente Colombia tiene 7,6 millones de conexiones a Internet de banda ancha. [1]. En el informe se observa que al finalizar el año 2013, Colombia registró 7.639.061 suscripciones a Internet de banda ancha Vive Digital, lo cual representó un crecimiento del $22 \%$ respecto al informe presentado en el mismo trimestre en el año 2012. Al principio del gobierno, 8 de cada 100 personas estaban suscritas al servicio de Internet, actualmente hay 16 por cada 100 personas [2]. Desde que se inició el gobierno y al cierre del año 2013 se registró un crecimiento de $182 \%$ en las conexiones a Internet Vive Digital. En la siguiente figura se muestran los suscriptores y penetración de banda ancha en el plan vive digital:

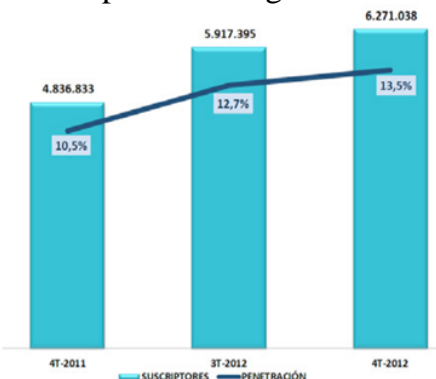

Fig. 1. Suscriptores y penetración Banda Ancha. Fuente: Ministerio TIC, Boletín trimestral de las TIC cifras tercer trimestre 2013.
También presentan en el informe un consolidado del crecimiento por departamentos de las conexiones a internet fijo residencial, donde se observa que aún quedan departamentos con una participación menor al 1\% como: Vichada, Guainía, Guaviare, Vaupés y Amazonas, que por sus condiciones geográficas especiales no son de fácil acceso para llevar internet por medio de tecnologías como cable o radioeléctricas.

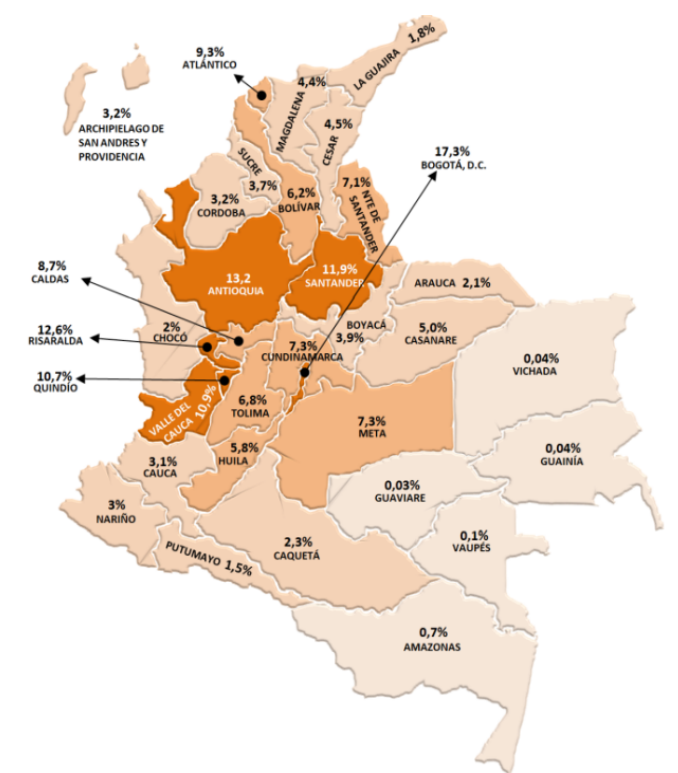

Fig. 2. Índice de penetración de internet fijo por departamentos y distrito capital.

Fuente: Ministerio TIC, Boletín trimestral de las TIC cifras tercer trimestre 2013.

\subsection{Dividendo digital}

Para lograr la conexión en la áreas mas remotas del país existe una iniciativa de utilizar la banda de 700 $\mathrm{MHz}$ a través del proyecto Dividendo Digital, que es el rango de espectro radioeléctrico UHF (Ultra High Frecuency) resultante de la transición de la televisión analógica a digital, el cual por sus ventajas en propagación de señal brinda la oportunidad de ampliar la cobertura de los servicios de banda ancha.

En la región 2 (ver figura 3) a la cual pertenece el territorio colombiano, la UIT (Unión Internacional de Telecomunicaciones) identifico la banda de 698 a $806 \mathrm{MHz}$, que corresponde a los canales $U H F$ del 52 al 69, equivalente a $108 \mathrm{MHz}$ de espectro como se observa en la Fig. 4; esto es explicado por el ANE (Agencia Nacional del Espectro) en el documento de consulta pública sobre las consideraciones técnicas en el uso de la banda del dividendo digital publicado el trece de enero de 2012 [3]. 


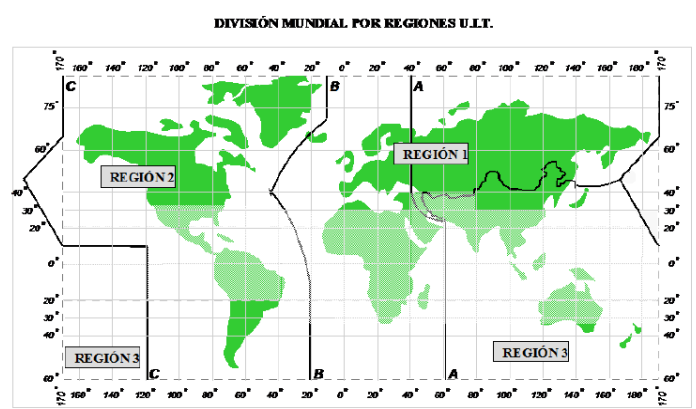

Fig. 3. División mundial por regiones U.I.T. Fuente: Ministerio TIC

Mediante la resolución 2623 de 2009 (de conformidad con la resolución 224 de la conferencia mundial de radiocomunicaciones CMR 2007), el ministerio TIC reservo "la banda de frecuencias de $698 \mathrm{MHz}$ a $806 \mathrm{MHz}$ a los servicios radioeléctricos fijo y móvil terrestre para proveer redes y servicios de telecomunicaciones que utilicen o lleguen a utilizar las IMT".

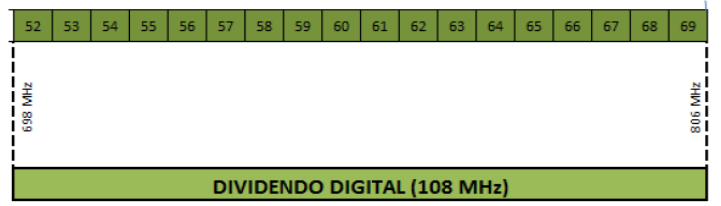

Fig. 4. Banda del dividendo digital para región 2. Fuente: ANE.

Esto con el fin de hacer uso eficiente del espectro disponible y así promover la expansión de la cobertura del internet móvil acorde con las iniciativas del plan vive digital, con el que se pretende llegar a 8,8 millones de conexiones a internet en el 2014 [4]. Debido a la importancia del uso del espectro $700 \mathrm{MHz}$ propuesto por el dividendo digital para expandir las conexiones a internet por medio de tecnologías inalámbricas, se hace necesario un estudio de los diferentes estándares que podrían ofrecer el servicio en dicha frecuencia de manera ágil y a muchos usuarios en las zonas rurales.

\section{ENTORNOS RURALES}

\subsection{LTE y WRAN}

Estos dos estándares funcionan bajo parámetros muy similares pero existen algunas diferencias que se observan en la Tabla 1.

El estándar LTE es un estándar global de comunicaciones móviles, creado por la $3 G P P$ ( $3 r d$ Generation Partnership Project) que desarrolló especificaciones del sistema de radio EPS (Evolved Packet System) y que junto a la arquitectura $S A E$ (System Architecture Evolution) [5] desarrollaron especificaciones para el funcionamiento de la 4 generación de redes móviles o 4G LTE. Esta red fue creada con el propósito de suplir la necesidad de los consumidores de grandes ciudades que exigen cada vez más una conexión estable y rápida. Teniendo eso en cuenta esta tecnología se centró en 3 pilares: cobertura, velocidad y respuesta.

Debido a que es un sistema de cobertura flexible le permite trabajar en casi cualquier frecuencia lo cual facilita que a frecuencias como la de $700 \mathrm{MHz}$ tenga una gran cobertura, también y gracias a su arquitectura basada en paquetes IP y pocos elementos de la red permite tiempos de respuesta o latencia más baja que sus antecesores $3 \mathrm{G}$ y $3.5 \mathrm{G}$ [6]. Gracias al uso y la implementación de tecnologías conjuntas como MIMO (Multiple-input Multiple-output) se logra aumentar la velocidad del sistema llegando a los $300 \mathrm{MB}$ en el enlace de bajada y a unos $86 \mathrm{MB}$ en el enlace de subida.

Por otro lado, el estándar WRAN (Wireless Regional Area Network) o 802.22 aparece con el propósito de brindar mayor cobertura y ampliar la actual capacidad. Se trata del primer estándar inalámbrico que implementa radio cognitiva. WRAN usa los espacios en blanco en las bandas de televisión y adicionalmente, a diferencia de LTE, emplea las técnicas de radio cognitiva ( $\mathrm{RC}$ ) para permitir la utilización compartida de espectro sin uso asignado a los servicios de radiodifusión de televisión [7].

Tabla 1. Parámetros LTE vs WRAN. Fuente: WRAN AND LTE COMPARISON IN RURAL

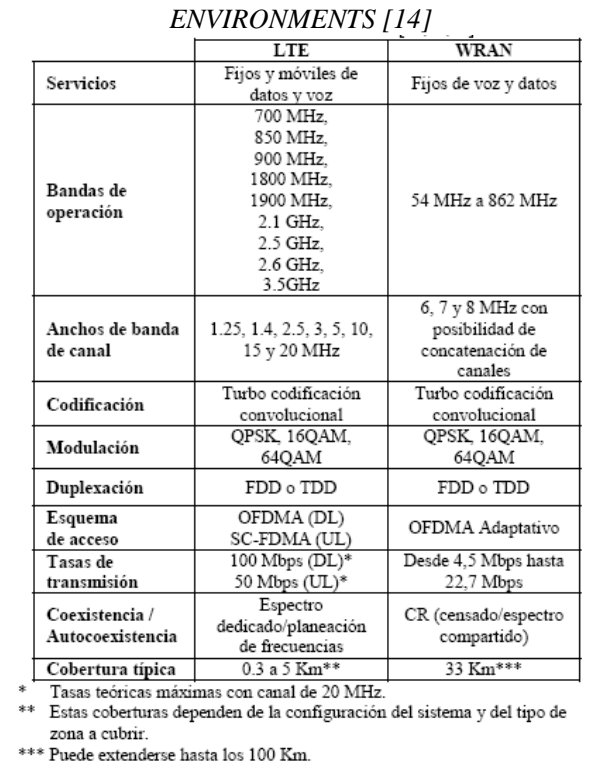


Esto se logra ya que RC identifica porciones del espectro que están inactivos, en un lugar y/o momento específico para el acceso oportuno de las frecuencias de televisión para la conectividad. Esta característica se conoce como re-configurabilidad, lo que es explicado en el estudio realizado en Zimbawe. [8]

$\mathrm{RC}$ es definido por la FCC (Federal Communications Commission) como un sistema de radio que lleva a cabo continuamente la detección del espectro, identifica dinámicamente espectro no utilizado y, a continuación opera en esos agujeros de espectro o espacios en blanco, donde los sistemas de radio con licencia (principal) no se activan. [9]

El estudio realizado en Malasya [7] afirma que esta iniciativa utiliza la base de no interferencia para permitir la conexión a Internet de banda ancha a las zonas rurales puesto que, puede brindar una mejor utilización de espectro ya que puede percibir su entorno y adaptarse a este. El uso de esta tecnología es la clave para este estándar (IEEE 802.22 WRAN) ya que no se requiere espectro dedicado, por lo tanto se ahorra en costos de licenciamiento. [8]

En el desarrollo del estudio llevado a cabo en Zimbawe [8] explican que el sistema es similar a $3 \mathrm{G}$, equipado con una antena direccional para la comunicación con la estación base y una antena omnidireccional para detectar y medir la condición del medio ambiente inalámbrico.

Adicionalmente, explican que tiene una estación base $(B S)$ que sirve de ubicación fija inalámbrica, que cuenta con un número de usuarios o equipos en las instalaciones del cliente $(C P E)$. Este $C P E$ es capaz de adaptarse y con el tiempo cambiar las características de transmisión como sea necesario para las actividades de los usuarios primarios.

También se controla el acceso al medio y se realizan funciones tradicionales como en los sistemas convencionales. [7]

\subsection{Simulación}

Teniendo en cuenta que existen varias simulaciones que representan el funcionamiento de LTE en la banda de los $700 \mathrm{MHZ}$, se realiza una simulación enfocada en WRAN sobre la misma banda con los siguientes parámetros:
Tabla 2. Parámetros simulación

\begin{tabular}{|l|r|}
\hline \multicolumn{1}{|c|}{ Parámetros } & Unidades \\
\hline Banda operación & $700 \mathrm{Mhz}$ \\
\hline Distancia & $33-100 \mathrm{Km}$ \\
\hline GanTx & $12 \mathrm{dBi}$ \\
\hline GanRx & $12 \mathrm{dBi}$ \\
\hline Ps EIRP & $36 \mathrm{dBm}$ \\
\hline Gr & $12 \mathrm{dBi}$ \\
\hline K & $1,38 \mathrm{E}-16 \mathrm{~J} / \mathrm{K}$ \\
\hline T & $300.15 \mathrm{Kelvin}$ \\
\hline Ancho Banda Canal & $6 \mathrm{Mhz}$ \\
\hline PotPromTx & $36 \mathrm{dBm}$ \\
\hline Celdas & 1 \\
\hline Distribución usuarios & Aleatoria \\
\hline HTML & $30 \mathrm{kbps} / 100 \mathrm{kbps}$ \\
\hline Real Time & $128 \mathrm{kbps} / 500 \mathrm{kbps}$ \\
\hline Streaming & $500 \mathrm{kbps} / 900 \mathrm{kbps}$ \\
\hline
\end{tabular}

El primer parámetro a considerar es la frecuencia (f), debido a que se está enfocado en entornos rurales se tomara como frecuencia $700 \mathrm{Mhz}$.

Ya definida la frecuencia se procede a calcular el Pathloss (PL: perdidas de propagación en $\mathrm{dB}$ ), los parámetros a considerar aquí son: distancia, frecuencia, ganancia transmisión, ganancia recepción.

La fórmula para el cálculo de $P L$ fue tomada de un trabajo realizado en la Universidad ICESI llamado Tecnología de canal de retorno para el estándar DVB-T mediante el uso de WRAN [10] y se determinó trabajar con el Modelo de propagación en espacio libre mejor conocido como modelo de Friis. La explicación del funcionamiento de este modelo se encuentra en [10].

$\mathrm{PL}=32.44+(20 * \log (\mathrm{d}))+(20 * \log (\mathrm{f}))-\mathrm{GanRx}-\mathrm{GanTx}$

Una vez calculado el PL, se calcula la interferencia. Donde Ps hace referencia a EIRP (Potencia radiada isotrópica efectiva) transmitida y Gr es la ganancia del receptor.

$\mathrm{I}=\mathrm{Ps}-\mathrm{PL}+\mathrm{Gr}$

Esta fórmula fue tomada de [7], estudio realizado en Malasia acerca de una posible implementación de WRAN. 
El valor de PS fue tomado de un documento elaborado por la Universidad Politécnica de Valencia [11] y el valor de Gr se tomó de [7].

Después se hallará el valor del ruido, fórmula utilizada en [12] donde los parámetros a considerar son la constante de Boltzman $(\mathrm{K}=1.3806504 \mathrm{e}$ 23), temperatura del área (en grados kelvin) y el ancho de banda (B).

$$
\text { RuidoRx }=10 * \log 10(\mathrm{~K} * \mathrm{~T} * \mathrm{~B})
$$

Posteriormente se calculara la SINR (Signal Interference + Noise Ratio) tomando como valor de la potencia promedio de transmisión $36 \mathrm{dBm}$ [11].

$$
\text { SINR }=\text { PotPromTx } /(\mathrm{I}+\text { RuidoRx })
$$

Finalmente, el cálculo de la capacidad se efectuará siguiendo la teoría de Shannon [13]:

$$
\mathrm{C}=\mathrm{B} * \log 2(1+\mathrm{SINR})
$$

Se calcula la capacidad para tres servicios determinados con $\mathrm{x}$ cantidad de usuarios. Los servicios son: HTML, Real Time y Streaming.

\subsection{Resultados}

Basados en los servicios básicos de uso diario en internet como lo son consultas en internet (HTML) servicios de comunicación por IP como Skype (REALTIME) y por último la comunicación con uso de video (Streaming) se realizan varias simulaciones para comprobar el comportamiento del sistema en cuanto a la capacidad que tendría en realizar estas tareas básicas encontrando los siguientes resultados:

La primera grafica obtenida de la simulación es la de capacidad contra distancia, donde se observa que si el CPE se encuentra muy apartado de la estación base se tendrá una interferencia mayor y así mismo una pérdida de banda ancha disponible, aunque observamos que debido a su gran cobertura la velocidad no se ve tan afectada cuando estamos en el rango de los 0 a $35 \mathrm{KM}$, teniendo tan solo una pérdida del $0.1 \mathrm{Mbs}$ en la capacidad.

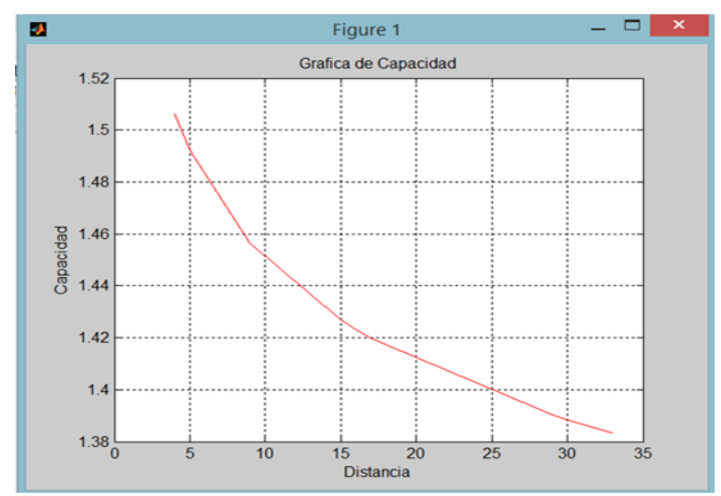

Fig. 5. Capacidad vs Distancia. Fuente: autores.

Después de ejecutar varias veces la simulación con números de usuarios aleatorios conectados, observamos que si los usuarios solo realizan consultas básicas (servicio HTML) sobre su conexión a internet como: consultar páginas web, bases de datos, bibliotecas o bancos, su consumo seria en promedio de 60 a $100 \mathrm{kbps}$ lo que representa tan solo un $18 \%$ del consumo total sobre su conexión.

Para facilitar el estudio del estándar nos basamos en la herramienta Skype la cual nos permite en tiempo real saber el consumo exacto por usuario dependiendo de lo que esté trabajando en el momento, teniendo esto presente observamos que: en el caso del servicio de Realtime este estándar permite el uso de la herramienta Skype para realizar una llamada con una calidad normal sin video entre dos participantes con total normalidad sin presentar interrupciones o pérdida del audio, también se puede compartir pantalla entre los participantes teniendo un consumo total sobre la banda ancha del CPE de $400 \mathrm{Kbs}$, lo cual representa un consumo del $33 \%$ sobre el CPE.

Por último, si los dos participantes realizan una video llamada en calidad normal (480p) se llegaría a ocupar hasta $900 \mathrm{kbs}$ de consumo en la banda ancha, y, si se realiza un stream de video como subir un video en vivo de un PC a youtube, entre otros, se ocuparía la totalidad de capacidad del CPE con sólo un usuario dejando lenta o casi nula la conexión para más solicitudes a internet. 


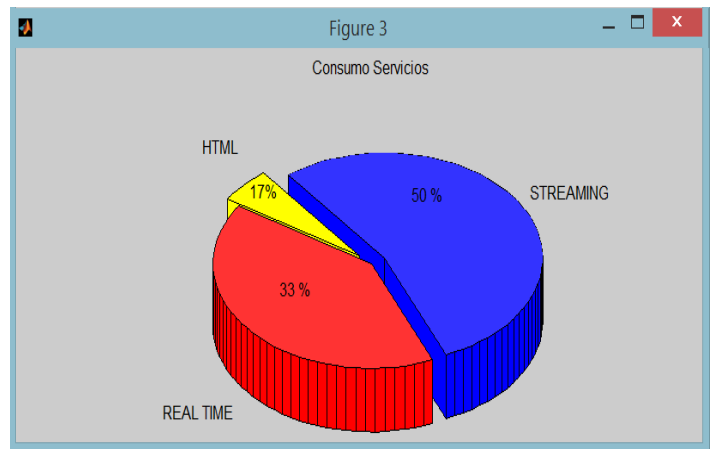

Fig. 6. Predicción de capacidad WRAN $700 \mathrm{MHz}$. Fuente: autores.

Por otro lado, para complementar este estudio se analizan las conclusiones presentadas en [14] donde realizan una comparación entre los dos estándares en los aspectos de cobertura y capacidad.

Hablando de cobertura se llega a la conclusión de que WRAN tiene mayor rango de cobertura con $29.75 \mathrm{Km}$ frente a los $25.1 \mathrm{Km}$ que ofrece LTE debido a que en este estándar el desempeño disminuye cuando los canales son mas angostos. [14].

\section{CONCLUSIONES}

Varios estudios, entre ellos el realizado por la Asociación GSM - GSMA, y Telecom Advisory Services LLC, donde evalúan el impacto cualitativo y cuantitativo que tendrían los usos alternativos del espectro del Dividendo Digital, llegan a la conclusión que asignar el espectro del Dividendo Digital a servicios de telecomunicaciones móviles terrestres en América Latina genera más beneficios económicos y sociales, que mantenerlo asignado a servicios de radiodifusión. [15]. Es por esto que el uso de la banda de los $700 \mathrm{MHz}$ planteado por el dividendo digital y el actual impulso que da el gobierno con sus programas de conexión para toda Colombia nos lleva a pensar en tecnologías que puedan cumplir con esa ambición.

En la actualidad Colombia pasa por una revolución tecnológica con la implementación de la cuarta generación de la tecnología móvil 4g LTE. Para poder ofrecer internet en todas esas zonas rurales de Colombia se firmó un acuerdo con los operadores dominantes en tecnología móvil para que ellos presten la infraestructura y lleven el servicio de internet a esas zonas, lo cual cumpliría con lo propuesto por el gobierno. Sin embargo, existen algunas limitaciones para llevar a cabo este despliegue, uno de ellos es la imposibilidad del sistema LTE para coexistir con otras tecnologías en el mismo espacio espectral ya que requiere espectro licenciado y contrario a WRAN no cuenta con la ventaja de utilizar radio cognitiva, además LTE no es un estándar diseñado para ambientes rurales lo cual se ve compensado por su alta transferencia de datos y a diferencia de WRAN es un estándar que ya ha sido implementado en muchas partes del mundo.

Las limitaciones que posee $L T E$ llevan a pensar en sistemas más dinámicos como WRAN, que gracias a la implementación de radio cognitiva en su funcionamiento permite su ejecución en la banda de los $700 \mathrm{MHz}$ sin que esta esté totalmente libre, adicionalmente no requiere espectro dedicado, por lo tanto se ahorra en costos de licenciamiento. A pesar de ser un estándar pensado específicamente para entornos rurales, no se encuentra ampliamente probado ni implementado, además la infraestructura para su funcionamiento aun nos se encuentra disponible para el uso comercial masivo lo que hace más compleja su implementación.

A pesar de los proyectos, iniciativas y objetivos que tiene trazados el gobierno a través del plan vive digital, $4 \mathrm{~g}$ para todos y el dividendo digital, aun quedaran zonas del país que a pesar de esas proyecciones no se verán cubiertas. Es en este escenario donde es importante considerar la implementación de WRAN para aquellas zonas rurales donde se necesite de una amplia cobertura y proporcionar un servicio básico de internet que complementaría y ayudaría a cumplir los objetivos proyectados por el gobierno.

\section{REFERENCIAS}

[1]. MinTIC, Colombia vive una revolución digital, Fecha de acceso: 22/03/2014, http://www.mintic.gov.co/portal/604/w3article-5143.html

[2]. MinTIC, Boletín trimestral de las TIC, Fecha de acceso: 22/03/2014, http://colombiatic.mintic.gov.co/602/articles4992_archivo_pdf.pdf

[3]. MinTIC. Documento de consulta pública sobre las consideraciones técnicas en el uso de la banda del dividendo digital., Fecha de acceso: 22/03/2014, http://www.mintic.gov.co/images/MS_ANE/ documento_de_consulta_publica_dividendo_ digital.pdf

[4]. MinTIC. Objetivos y aspiraciones del Plan Vive Digital, Fecha de acceso: 22/03/2014, 
http://www.mintic.gov.co/index.php/vivedigital-plan/objetivos-aspiraciones

[5]. 3GPP, The motivation for LTE. Fecha de acceso: 22/03/2014, http://www.3gpp.org/technologies/keywordsacronyms/98-lte

[6]. 3GGP, 4G Mobile Broadband Evolution: $3 G P P$ release 10 and Beyond, Fecha de acceso: 22/03/2014, http://www.4gamericas.org/documents/4G\% 20Americas_3GPP_Rel10_Beyond_2.1.11\%20.pdf

[7]. Hasbullah Omar, Suhaidi Hassan y Ahmad Hanis, "Feasibility Study of Using IEEE 802.22 Wireless Regional Area Network (WRAN) in Malaysia", 2010.

[8]. Mardeni Roslee y Chimheno Rasty, "WRAN System on ZBC TV: A New Way to Internet Connectivity for Rural Areas in Zimbabwe", 2012.

[9]. W. L. Chin, H. C. Kuo y H. Chen, "Features detection assisted spectrum sensing in wireless regional area network cognitive radio systems", 2010.

[10]. Universidad ICESI, Tecnología de canal de retorno para el estándar DVB-T mediante el uso de WRAN, Fecha de acceso: 22/03/2014, http://bibliotecadigital.icesi.edu.co/biblioteca _digital/bitstream/10906/68395/1/tecnologia _canal_retorno.pdf
[11]. Universidad Politécnica de Valencia, El estándar IEEE-802.22 Wireless Regional Area Network (WRAN), Fecha de acceso: 22/03/2014,

http://iba.dit.upm.es/w/images/7/7f/El_estan dar_IEEE-802.22.pdf

[12]. Daniel Orlando Bejarano Gonzalez, “Diseño y desarrollo de un software para simulación de propagación en redes LTE.”, Tesis de Pregrado, facultad de Ingeniería de telecomunicaciones, Universidad Piloto de Colombia, Bogotá, Colombia, 2012.

[13]. Vodafone, LTE: Nuevas Tendencias en Comunicaciones Móviles, Fecha de acceso: 22/03/2014,

http://www.vodafone.es/static/fichero/pre_uc m_mgmt_002620.pdf

[14]. Herrera O.E., Alejandro Gutiérrez, Ana Ospina y Alexander Galvis, "WRAN and LTE comparison in rural environments", 2012, Communications Conference (COLCOM), 2012 IEEE Colombian. DOI: 10.1109/ColComCon.2012.6233675

[15]. ANE, Documento de consulta pública sobre las consideraciones técnicas en el uso de la banda del dividendo digital, Fecha de acceso: 22/03/2014, http://www.mintic.gov.co/images/MS_ANE/ documento_de_consulta_publica_dividendo_ digital.pdf 